\title{
Exosomal transfer of circular RNA FBXW7 ameliorates the chemoresistance to oxaliplatin in colorectal cancer by sponging miR-18b-5p
}

\author{
Yeqing XU11, Aizhu QIU², Feng PENG ${ }^{1}$, Xiangyun TAN ${ }^{1}$, Jin WANG ${ }^{1}$, Xiaosong GONG ${ }^{1, *}$ \\ ${ }^{1}$ Department of General Surgery, The Affiliated Zhuzhou Hospital Xiangya Medical College CSU, Zhuzhou, Hunan, China; ${ }^{2}$ Department of \\ Basic Medicine, Hunan Traditional Chinese Medicine College, Zhuzhou, Hunan, China
}

${ }^{*}$ Correspondence: biyahuan19780616@163.com

Received April 17, 2020 / Accepted July 21, 2020

\begin{abstract}
Circular RNA F-box and WD repeat domain containing 7 (circ-FBXW7) has been revealed to be involved in the tumorigenesis of colorectal cancer (CRC). Exosomes are critical mediators of intercellular communication. However, the role of exosomal circ-FBXW7 in the CRC oxaliplatin resistance remains unknown. Cell viability, apoptosis, motility, and drug efflux were measured by the cell counting kit- 8 assay, flow cytometry, transwell assay, and atomic absorption spectrophotometry, respectively. The expression of circ-FBXW7 and microRNA (miR)-18b-5p was detected using the quantitative realtime polymerase chain reaction. Western blot was used to determine multidrug resistance protein 1 (MRP1), myeloid cell leukemia-1 (MCL-1), CD9, CD63, Caspase3, E-cadherin, and N-cadherin. Exosomes were isolated and captured using the ultracentrifugation method and transmission electron microscopy. The interaction between circ-FBXW7 and miR-18b-5p was confirmed by dual-luciferase reporter assay and RNA immunoprecipitation assay. In vivo experiments were conducted using the murine xenograft model. Our results showed that circ-FBXW7 was decreased in oxaliplatin-resistant CRC patients and cells. circ-FBXW7 was secreted by circ-FBXW7-transfected FHC cells and could be transferred to resistant CRC cells through the exosome secretion. Subsequently, in vitro and in vivo studies demonstrated exosomal circ-FBXW7 led resistant cells sensitive to oxaliplatin, increased the oxaliplatin-induced apoptosis, inhibited oxaliplatin-induced epithelial-mesenchymal transition, and suppressed oxaliplatin efflux. miR-18b-5p was increased in oxaliplatin-resistant CRC patients and cells and was confirmed to be a target of circ-FBXW7. Immediately, the rescue assay showed exosome-mediated transfer of circ-FBXW7 enhanced oxaliplatin sensitivity by binding to miR-18b-5p in vitro and in vivo. To conclude, the circ-FBXW7 delivery by exosomes could ameliorate chemoresistance to oxaliplatin in CRC by directly binding to miR-128-3p, suggesting a promising therapeutic strategy for oxaliplatin-resistant CRC patients.
\end{abstract}

Key words: circ-FBXW7, miR-18b-5p, oxaliplatin, exosome, colorectal cancer

Colorectal cancer (CRC) is the third most common solid malignancy and the fourth-highest leading cause of cancer-related mortality worldwide [1]. With the improvement in early diagnosis and multimodality treatment, the average survival time of advanced CRC has roughly doubled; however, the patients still die within three years $[2,3]$. Currently, oxaliplatin-based chemotherapy is one of the therapeutically efficacious strategies for CRC therapy, however, obvious resistance to oxaliplatin has been emerged in CRC, thereby leading to treatment failure [4]. Therefore, extensive efforts on developing novel therapeutic approaches for CRC chemoresistance are required.

Exosomes are extracellular vesicles with a diameter ranging from $50 \mathrm{~nm}$ to $100 \mathrm{~nm}$ and can be secreted by a wide range of cell types, including cancer cells [5]. Exosomes are considered as mediators of tumor communication that can transfer and exchange their cargoes, such as protein, DNA, microRNA (miRNA), mRNA, and circular RNA (circRNA), between tumor cells and extracellular microenvironment [6-8], thereby performing various functions, such as the regulation of immune response [9], cell metabolism [10], metastasis, angiogenesis, and the conference of drug resistance [11] in cancers. Moreover, exosomes relative stably present in almost circulating body fluid, such as blood, urine, saliva, cerebrospinal fluid, etc., and can transit biological barriers, like the blood-brain barrier [12]. Based on these features, it has been suggested that exosomes will be useful for the noninvasive diagnostic and therapeutic of cancers [13].

circRNAs are one of the conserved RNA transcripts with covalently closed loop structures, which make them resistant to regular mechanisms of linear RNAs decay [14]. Increasing 
evidence has revealed that circRNAs play important roles in many cancers by involving in the regulation of cell growth, differentiation, metastasis, apoptosis, and drug resistance $[15,16]$. Recently, circRNAs acted as one of the novel objects that have also been evaluated in CRC and many circRNAs, like circRNA102958 [17], circ-ITGA7 [18], were reported to be implicated in the tumorigenesis and progression of CRC. CircRNAs may be a potential therapeutic target for CRC. CircRNA-F-box and WD repeat domain containing 7 (circFBXW7) is a novel functional circRNA and has been recently recognized as a tumor suppressor to suppress tumorigenesis and progression in CRC [19]. Therefore, we assumed that circ-FBXW7 might regulate drug resistance and cell progression in CRC.

In this study, we attempted to evaluate the roles as well as the underlying molecular mechanism of exosome-transmitted circ-FBXW7 in the oxaliplatin-resistant in CRC, which may shed light on the development of potential therapy for oxaliplatin-resistant CRC.

\section{Patients and methods}

Patients and specimens. A total of 56 CRC tissues were obtained from patients who underwent surgical resection at the Affiliated Zhuzhou Hospital Xiangya Medical College CSU and were stored at $-80^{\circ} \mathrm{C}$ until used. All patients were diagnosed by histopathological examination and only received oxaliplatin-based neoadjuvant chemotherapy prior to surgery. The patients were classified into oxaliplatinresistant group (resistant-CRC, $\mathrm{n}=30$ ) and -sensitive group (sensitive-CRC, $\mathrm{n}=26$ ). Whether the patients were sensitive to oxaliplatin was screened according to radiology and solid tumor response assessment, patients with symptoms worsening, new lesions emergence, or $\geq 25 \%$ of tumors regenerating on imaging evaluation are oxaliplatin-resistant patients, otherwise sensitive patients. This study was permitted by the Ethics Committee of the Affiliated Zhuzhou Hospital Xiangya Medical College CSU and written informed consent was collected from all subjects.

Cell culture. Human CRC cell line (SW480 and HCT116), normal colon (FHC) cells, and 293T cells were obtained from the Shanghai Academy of Life Science (Shanghai, China) and maintained in the Dulbecco's modified Eagle's medium (DMEM; Gibco, Carlsbad, CA, USA) supplemented with 10\% fetal bovine serum and 1\% penicillin/streptomycin (Gibco) in $5 \% \mathrm{CO}_{2}$ at $37^{\circ} \mathrm{C}$. SW480 and HCT116 cells were used to generate oxaliplatin (oxa) resistant cells named SW480/OxR and HCT116/OxR as previously described [20]. For exosome co-cultures, $2 \mu \mathrm{g} / \mathrm{ml}$ of exosomes were incubated with the recipient cells $\left(6 \times 10^{5}\right)$ in the culture medium.

Cells viability assay. Cell Counting Kit-8 (CCK-8) assay was used to analyze cell viability. The cells were seeded into 96-well plates at a density of 5,000 cells/well and treated with oxaliplatin at indicated concentrations. After that, $10 \mu \mathrm{l}$ CCK-8 solution was added to each well for $2 \mathrm{~h}$. Subsequently, the optical density (OD) at $450 \mathrm{~nm}$ was determined by a microplate reader at the indicated time points. Besides that, the half-maximal inhibitory concentration (IC50) value of drugs was assessed according to the relative survival curve.

Cell apoptosis assay. Apoptotic cells were measured using Annexin V-fluorescein isothiocyanate (FITC)/propidium iodide (PI) apoptosis detection kit (BD Biosciences, San Jose, CA, USA) following the standard protocol. In brief, cells treated with $30 \mu \mathrm{M}$ oxaliplatin were resuspended with binding buffer and then stained with $5 \mu$ FITC annexin V and $10 \mu \mathrm{l}$ PI. Finally, the apoptotic rate was analyzed by FlowJo software.

Cell migration and invasion assays. For migration assay, cells were seeded on the top chambers with serum-free DMEM. Then the lower chambers were filled with serumcontaining DMEM. After $24 \mathrm{~h}$ incubation, cells on the lower face of the membranes were fixed and stained. Finally, migrated cells were counted with a microscope. For invasion assay, the top chambers were pre-coated with the matrigel (BD Biosciences) and the rest of the experiment was similar to the steps of cell migration.

Total cellular oxaliplatin and DNA-bound quantitative platinum (Pt) assay. For the content of intracellular Pt, cells treated with $30 \mu \mathrm{M}$ oxaliplatin for $24 \mathrm{~h}$ were lysed using $0.1 \%$ Triton-X-100 and $0.2 \%$ nitric acid overnight. Next, the lysate was analyzed using atomic absorption spectrometry and the protein was quantified by a BCA method. For DNA-binding Pt content, DNeasy Blood \& Tissue Kits (Qiagen, Valencia, CA, USA) was used to isolate DNA following the standard protocol, and then the isolated DNA was determined by the NanoDrop spectrophotometer. Finally, the same DNA hydrolysate was sent for $\mathrm{Pt}$ analysis using atomic absorption spectrometry.

Quantitative real-time polymerase chain reaction (qRT-PCR). Total RNA was exacted using miRNeasy Micro Kit (Qiagen) from cells and exosomes. Then total RNA was reversely transcribed into complementary DNA (cDNA) using the Prime Script RT Master Mix (Applied Biosystems, Foster City, CA, USA). After that, qRT-PCR was performed with SYBR-Green PCR Master Mix Kit (Qiagen). Fold changes were analyzed by the $2^{-\Delta \Delta C t}$ method and normalized by glyceraldehyde 3-phosphate dehydrogenase (GADPH) or U6 small nuclear B noncoding RNA (U6). The specific primer sequences were presented as follows: circ-FBXW7: $\mathrm{F}$ 5'-ACTGGAAAGTGACTCTGGGA-3', R 5'-TACTGGGGCTAGGCAAACAA-3'; miR-18b-5p: F 5'-TGTGCAAATCCATGCAAAACTGA-3', R 5'-GTGCAGGGTCCGAGGT-3'; GADPH: F 5'-GATATTGTTGCCATCAATGAC-3', R 5'-TTGATTTTGGAGGGATCTCG-3'; U6: F 5'-CTCGCTTCGGCAGCACA-3', R 5'-ACGCTTCACGAA-TTTGCGT-3'.

Cell transfection. The miR-18b-5p mimic and negative control (miR-NC) were purchased from RIBOBIO (Guangzhou, China). The empty vector (Vector), pcDNA3.1-CircFBXW7 overexpression vector (Circ-FBXW7) were synthesized by Genepharma (Shanghai, China). The transfection of 
oligonucleotides was performed using Lipofectamine ${ }^{\mathrm{Tw}} 3000$ transfection reagent (Invitrogen, Carlsbad, CA, USA).

Exosome (exo) isolation. Exosomes were isolated from FHC cell culture media by the ultracentrifugation method. Cell culture fluid was centrifuged at $3,000 \mathrm{~g}$ for $30 \mathrm{~min}$ at $4{ }^{\circ} \mathrm{C}$ to remove cellular debris/dead cells. Next, the resulting supernatant was further centrifuged at $100,000 \mathrm{~g}$ for $70 \mathrm{~min}$ at $4{ }^{\circ} \mathrm{C}$ and followed by filtering using $0.22 \mu \mathrm{m}$ filtration to concentrate the exosome-containing solution. After washing with PBS, the supernatant was centrifuged at $100,000 \mathrm{~g}$ for 70 min again. Finally, cell exosomes were collected.

Transmission electron microscopy (TEM). $10 \mathrm{ml}$ exosome pellets were dropped on the carbon-coated copper grid and incubated for $5 \mathrm{~min}$ at $37^{\circ} \mathrm{C}$, and then subjected to $2 \%$ phosphotungstic acid solution for $2 \mathrm{~min}$. After washing with PBS 3 times, the prepared samples were captured using a transmission electron microscope (JEOL, Akishima, Japan).

Electroporation of circ-FBXW7 into exosomes. circFBXW7 was encapsulated in exosomes using the Gene Pulser X Electroporator (Bio-Rad, Hercules, CA, USA). In brief, $50 \mathrm{nmol}$ circ-FBXW7 overexpression vector and $10 \mu \mathrm{g}$ exosomes were gently mixed in $200 \mu$ lelectroporation buffer at $4^{\circ} \mathrm{C}$. After electroporation at $350 \mathrm{~V}$ and $150 \mu \mathrm{F}$, the mixture was incubated for $30 \mathrm{~min}$ at $37^{\circ} \mathrm{C}$ to ensure the recovery of exosome membranes. Recovery was determined by TEM as described above.

Western blot. Proteins were extracted using RIPA lysis buffer (Beyotime, Beijing, China) and quantified by the bicinchoninic acid (BAC) method following the standard protocol. Then extracted proteins were separated on sodium dodecyl sulfate-polyacrylamide gel electrophoresis, transferred onto a polyvinylidene fluoride membrane, and blocked with 5\% non-milk. After that, the membrane was incubated with primary antibodies against multidrug resistance protein 1 (MRP1) (1:1000, ab233383, Abcam, Cambridge, MA, USA), myeloid cell leukemia-1 (MCL-1) (1:5000, ab32087, Abcam), CD9 (1:5000, ab68418, Abcam), CD63 (1:2000, ab68418, Abcam), cleaved caspase-3 (1:1000, ab13847, Abcam), E-cadherin (E-cad) (1:1000, ab15148, Abcam), $\mathrm{N}$-cadherin (N-cad) (1:1000, ab18203, Abcam), and GAPDH (1:10000, ab181602, Abcam), followed by the incubation with HRP-conjugated secondary antibody (1:1000, ab9482, Abcam). The protein bands were visualized using the chemiluminescence chromogenic substrate (Beyotime).

Dual-luciferase reporter assay. The circ-FBXW7 possessing wild-type (WT) or mutant (MUT) binding sequences of miR-18b-5p was cloned into the pmiR-RBReport (Promega, Shanghai, China), respectively. Subsequently, these constructed vectors were co-transfected into SW480 and HCT116 cells with miR-18b-5p mimics or miR-NC using Lipofectamine 3000 (Invitrogen). Lastly, a dual-luciferase assay kit (Promega) was used to detect the luciferase activity.

RNA immunoprecipitation (RIP) assay. RIP assay was performed using the Magna RNA immunoprecipitation kit
(Millipore, Billerica, MA, USA). SW480 and HCT116 cells were lysed in RIP buffer and then the lysate was incubated with magnetic beads coated with anti-Ago 2 or IgG antibody. Finally, purified RNA was analyzed by qRT-PCR.

Xenograft experiments in vivo. Five-week-old athymic $\mathrm{BALB} / \mathrm{c}$ nude mice $(\mathrm{n}=18)$ were obtained from Jinan Pengyue Animal Center (Jinan, China). HCT116/OxR cells were subcutaneously inoculated into the flanks of the nude mice. After inoculation for 7 days, the same amounts of Vectorexo, circ-FBXW7-exo, or PBS were intratumorally injected with oxaliplatin twice weekly. Subsequently, the tumor size in mice was detected every week. The mice were sacrificed after 7 weeks, and the tumor masses were weighted and harvested for further molecular analysis. The animal experiments were approved by the Ethics Committee of the Affiliated Zhuzhou Hospital Xiangya Medical College CSU.

Statistical analysis. Data were expressed as the mean \pm standard deviation (SD) and analyzed by GraphPad Prism 7 software (GraphPad Inc., San Diego, CA, USA). Significant differences between different groups were analyzed using Student's t-test or one-way analysis of variance (ANOVA). The correlation analysis was performed using Spearman's correlation test. A p-value $<0.05$ suggested a statistical significance.

\section{Results}

Oxaliplatin resistance promotes cell viability, invasion, migration, drug efflux, and suppresses cell apoptosis in CRC. The influences of oxaliplatin resistance on CRC cells were detected. Results exhibited that resistant cells had a poor response to oxaliplatin compared with parental cells,

Table 1. Clinicopathological characteristics of patients in the study.

\begin{tabular}{|c|c|c|c|c|}
\hline \multirow[b]{2}{*}{ Characteristics } & \multirow[b]{2}{*}{$\mathbf{n}$} & \multicolumn{2}{|c|}{ Patients } & \multirow[b]{2}{*}{ p-value } \\
\hline & & $\begin{array}{c}\text { Sensitive } \\
(\mathrm{n}, \%)\end{array}$ & $\begin{array}{c}\text { Resistant } \\
(\mathrm{n}, \%)\end{array}$ & \\
\hline \multicolumn{5}{|l|}{ Age (years) } \\
\hline$<60$ & 23 & 9 & 14 & 0.4216 \\
\hline$\geq 60$ & 33 & 17 & 16 & \\
\hline \multicolumn{5}{|l|}{ Gender } \\
\hline Male & 27 & 12 & 15 & 0.7952 \\
\hline Female & 29 & 14 & 15 & \\
\hline \multicolumn{5}{|l|}{ TNM stage } \\
\hline $\mathrm{I}+\mathrm{II}$ & 31 & 20 & 11 & $0.0033^{*}$ \\
\hline $\mathrm{III}+\mathrm{IV}$ & 25 & 6 & 19 & \\
\hline Lymph node metastasis & & & & $0.0005^{*}$ \\
\hline Yes & 39 & 12 & 27 & \\
\hline No & 17 & 14 & 3 & \\
\hline Tumor recurrence & & & & $0.0426^{*}$ \\
\hline Yes & 40 & 15 & 25 & \\
\hline No & 16 & 11 & 5 & \\
\hline \multicolumn{5}{|l|}{ Circ-FBXW7 expression } \\
\hline High & 32 & 8 & 24 & $0.0003^{*}$ \\
\hline Low & 24 & 18 & 6 & \\
\hline
\end{tabular}


A
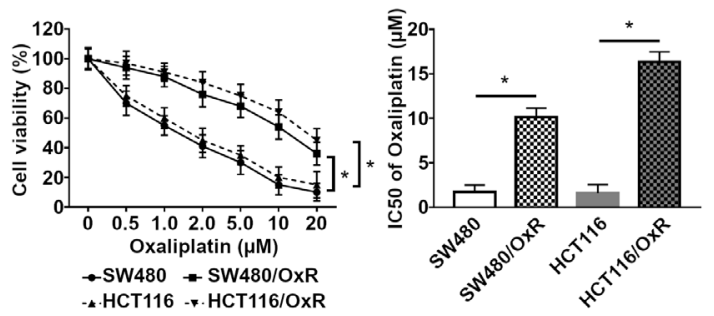

B
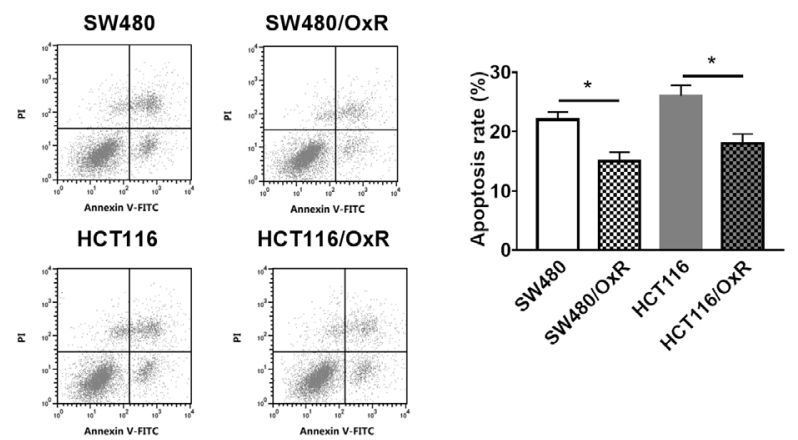

C
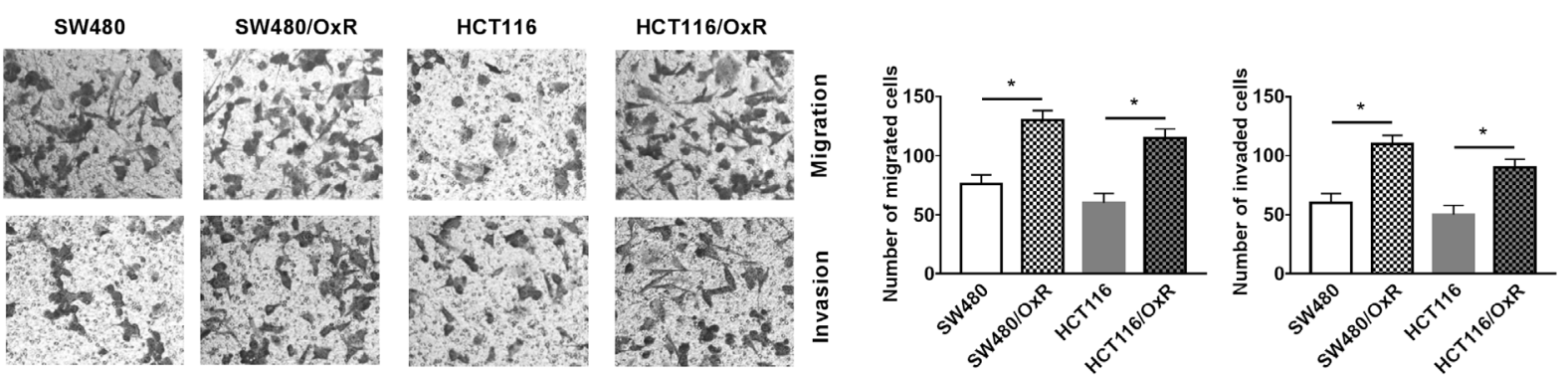

D

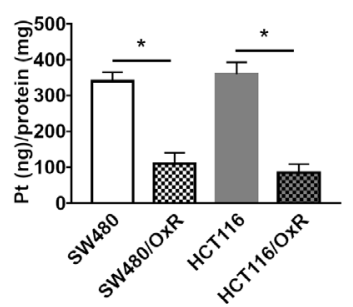

E

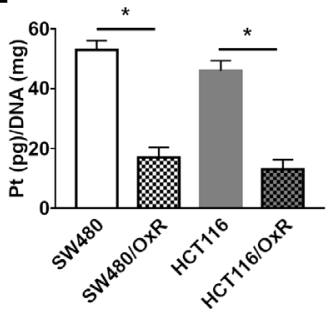

Figure 1. Oxaliplatin resistance promotes cell viability, invasion, migration, drug efflux, and suppresses cell apoptosis in CRC. A) The CCK-8 assay was used to detect cell viability and IC50 in parental and resistant cells in the treatment of oxaliplatin at indicated concentrations. B) The apoptosis of parental and resistant cells treated with $30 \mu \mathrm{M}$ oxaliplatin was analyzed using flow cytometry. C) The migration and invasion abilities in parental and resistant CRC cells were determined by the transwell assay. D) The accumulation of Pt was examined in parental and resistant cells after treatment with $30 \mu \mathrm{M}$ oxaliplatin for $24 \mathrm{~h} .{ }^{*} \mathrm{p}<\mathbf{0 . 0 5}$

reflected by the enhancement of cell viability and IC50 (Figure 1A) as well as the decrease of drug-induced apoptotic cells (Figure 1B). Moreover, SW480/OxR and HCT116/OxR showed high migration and invasion compared to their parental cells (Figure 1C). In the meanwhile, Pt analysis in cells treated with $30 \mu \mathrm{M}$ oxaliplatin for $24 \mathrm{~h}$ suggested total intracellular Pt and DNA-bound Pt was significantly lower in resistant cells than in parental cells (Figures 1D, 1E).

circ-FBXW7 is secreted by circ-FBXW7-transfected FHC cells and can be transferred to resistant CRC cells through exosome secretion. The previous study has reported circ-FBXW7 to be involved in the tumorigenesis of CRC [19], and cell-secreted exosomes and their contents can be internalized by surrounding cells [21]. Herein, we investigated the biological function of exosome-transmitted circ-FBXW7 in CRC cell oxaliplatin resistance. Firstly, the expression of circ-FBXW7 was measured and results showed circ-FBXW7 was lower in oxaliplatin-resistant CRC patients and resistant CRC cells than that in controls (Figures 2A, 2B). Moreover, the chemoresistance to oxaliplatin in CRC was correlated with advanced TNM stages, lymph node metastasis, tumor recurrence, and higher circ-FBXW7 expression (Table 1). Subsequently, FHC cells were transfected with circ-FBXW7 or Vector, and a significant increase of circ-FBXW7 expression was observed in circ-FBXW7-transfected FHC cells (Over-Circ-FBXW7-FHC) compared to vector-transfected FHC cells (Vector-FHC) (Figure 2C). After that, exosomes in the culture supernatant of these transfected FHC cells, named circ-FBXW7-exo and Vector-exo, were extracted. TEM analysis showed the morphology (round-shaped vesicles) and size $(100 \pm 60 \mathrm{~nm})$ of exosomes, and western blot showed the enrichment of exosomal markers CD9 and CD63 in exosomes (Figure 2D), suggesting the successful isolation of exosomes from the cell culture supernatant. What's more, the 
A
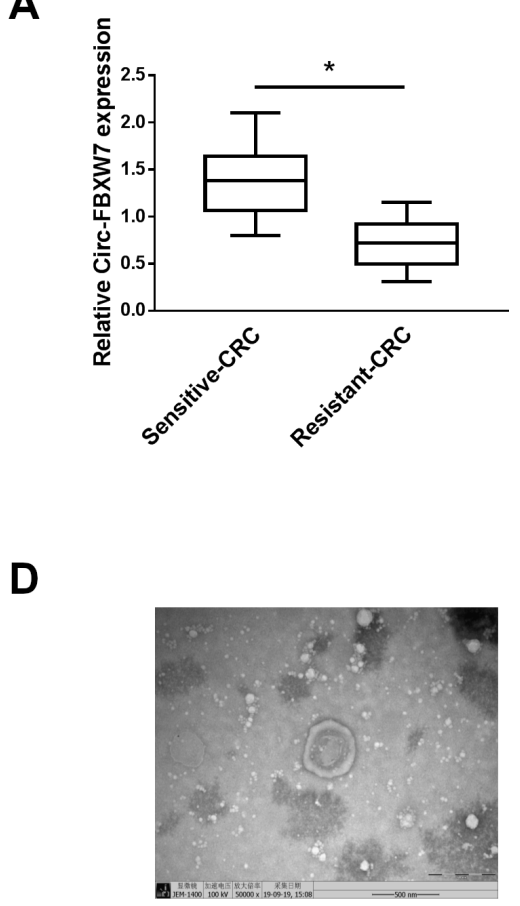

$\mathbf{F}$

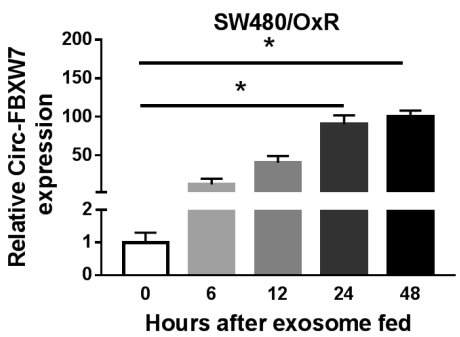

B
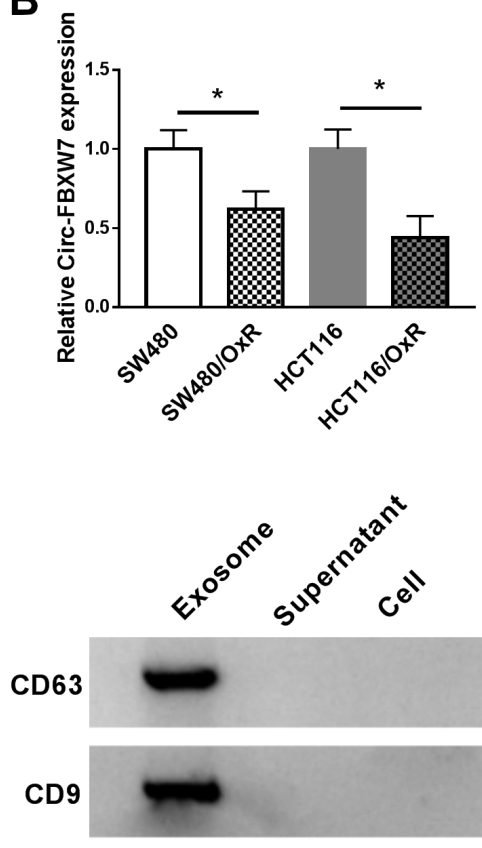

E

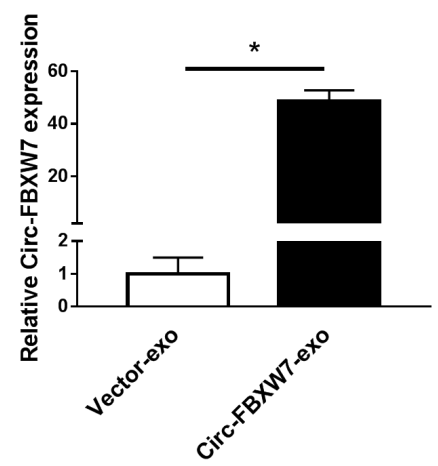

Figure 2. circ-FBXW7 is secreted by circ-FBXW7-transfected FHC cells and can be transferred to resistant CRC cells through exosome secretion. A, B) The expression of circ-FBXW7 in oxaliplatin-resistant and oxaliplatin-sensitive CRC patients, as well as in parental and resistant CRC cells was detected using qRT-PCR. C) The expression of circ-FBXW7 in FHC cells transfected with circ-FBXW7 or Vector was detected using qRT-PCR. D) The image of exosomes was captured by TEM. D) The expression levels of exosomal markers CD9 and CD63 were measured by western blot. E) The levels of circ-FBXW7 in exosomes derived from FHC cells transfected with circ-FBXW7 or Vector were determined using qRT-PCR. F) qRT-PCR was performed to analyze the level of circ-FBXW7 in resistant CRC cells co-maintained with different incubation times of circ-FBXW7-exo. ${ }^{\star}$ p $<0.05$

expression of circ-FBXW7 was higher in exosomes isolated from Over-Circ-FBXW7-FHC cells than that isolated from Vector-FHC cells (Figure 2E). After that, SW480/OxR and HCT116/OxR cells were co-cultured with circ-FBXW7-exo, we observed that circ-FBXW7 was obviously upregulated in resistant cells after co-incubation with circ-FBXW7-exo; besides, a corresponding increase of circ-FBXW7 expression in SW480/OxR and HCT116/OxR cells was found with the prolonged incubation (Figure $2 \mathrm{~F}$ ). Altogether, we confirmed Over-Circ-FBXW7-FHC cells could effectively secrete exosomes harboring circ-FBXW7, which were able to transfer to CRC resistant cells.
Intercellular transfer of circ-FBXW7 by exosomes enhances oxaliplatin sensitivity in vitro. We further explored whether exosome-transferred circ-FBXW7 could regulate chemoresistance in resistant cells. Subsequently, we discovered exosome-transferred circ-FBXW7 led SW480/OxR and HCT116/OxR cells to be sensitive to oxaliplatin, as illustrated by the decrease of cell viability (Figure 3A) and drug-resistance associated protein expression of MRP-1 and MCL-1 in resistant cells (Figure 3G), as well as by the increase of drug-induced apoptotic cells (Figure 3B) and cleaved caspase-3 protein (Figure 3G). Moreover, circ-FBXW7-exo inhibited the migration 


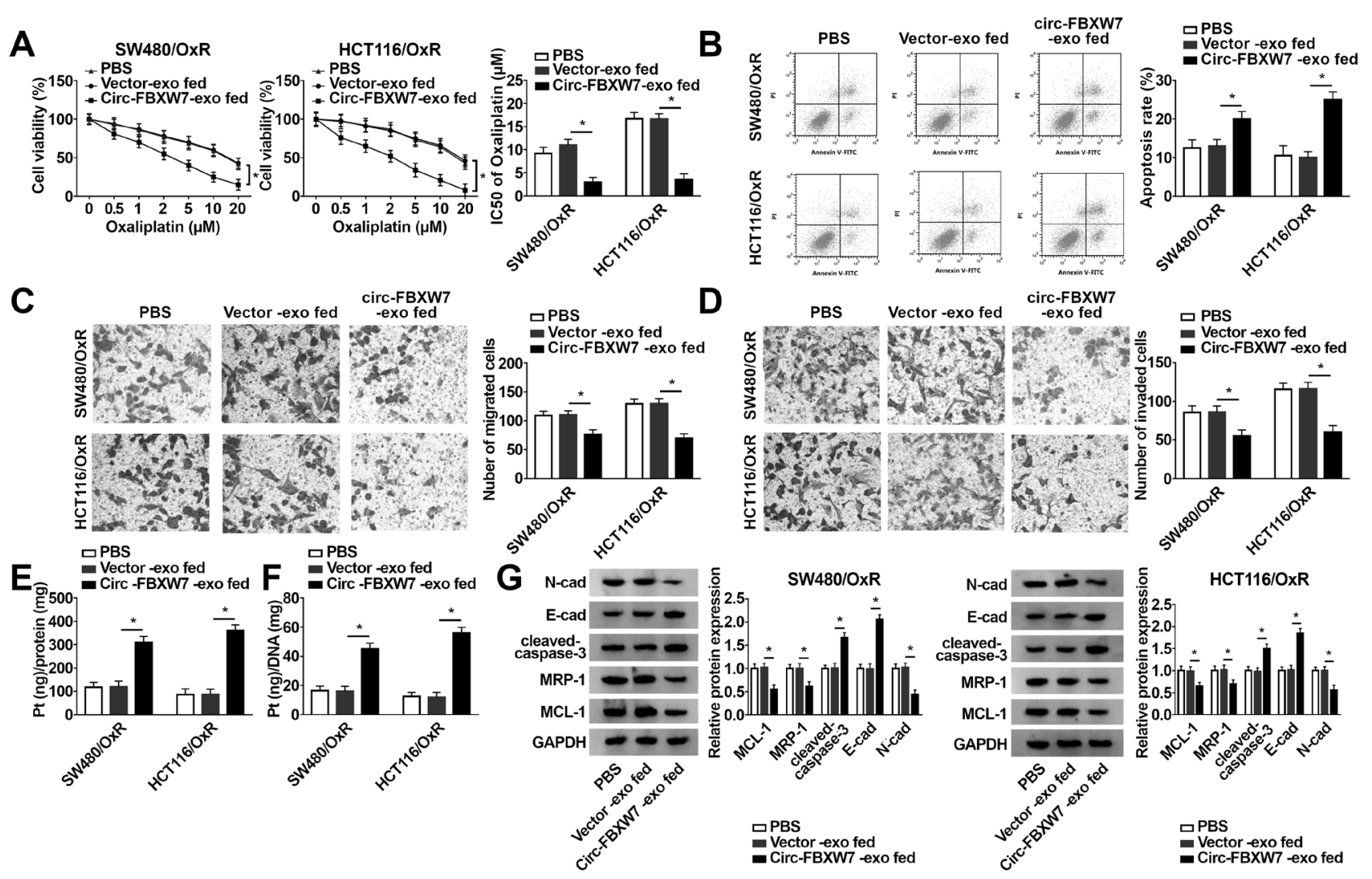

Figure 3. Intercellular transfer of circ-FBXW7 by exosomes enhances oxaliplatin sensitivity in vitro. circ-FBXW7 was transferred into the SW480/ OxR and HCT116/OxR cells by exosomes. A) Cell viability was detected using the CCK-8 assay in resistant cells treated with oxaliplatin at indicated concentrations. B) The apoptosis of resistant cells treated with $30 \mu \mathrm{M}$ oxaliplatin was analyzed using flow cytometry. Migration (C) and invasion (D) of SW480/OxR and HCT116/OxR cells were examined using the transwell assay. E, F) The accumulation of Pt was analyzed in resistant cells treated with $30 \mu \mathrm{M}$ oxaliplatin for $24 \mathrm{~h}$. G) Western blot was used to measure the level of $\mathrm{N}$-cad, E-cad, cleaved caspase-3, MRP-1, and MCL-1. ${ }^{\star} \mathrm{p}<0.05$

A

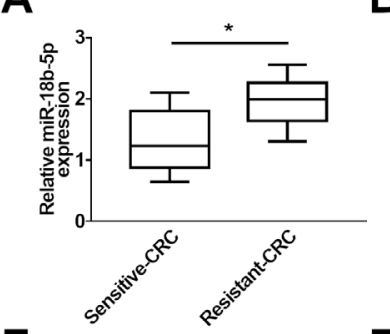

E

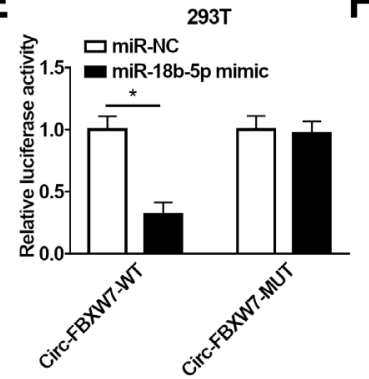

B

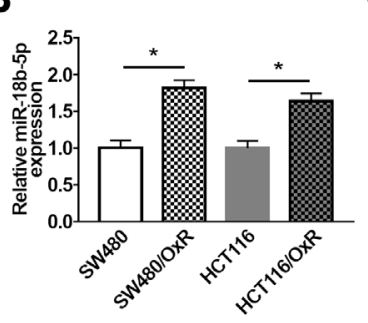

$\mathbf{F}$

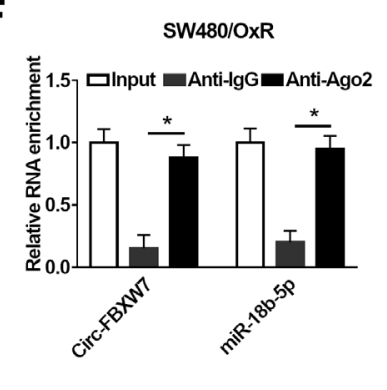

C

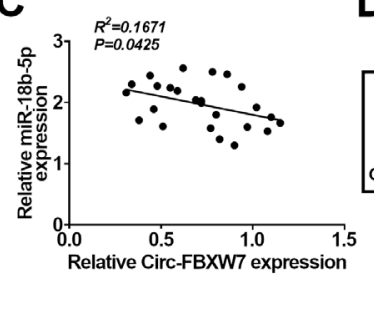

D

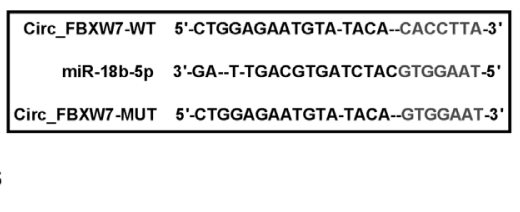

Figure 4. circ-FBXW7 is a sponge of miR-18b-5p in CRC cells. A, B) The expression of miR-18b-5p in oxaliplatin-resistant and oxaliplatin-sensitive CRC patients, as well as in parental and resistant CRC cells was detected using qRT-PCR. C) The correlation between miR-18b-5p and circ-FBXW7 was analyzed using Spearman's correlation test. D) The putative binding site between miR-18b-5p and circ-FBXW7 was presented. E, F) The interaction between miR-18b-5p and circ-FBXW7 was confirmed using the dual-luciferase reporter assay and RIP assay. ${ }^{*} \mathbf{p}<0.05$

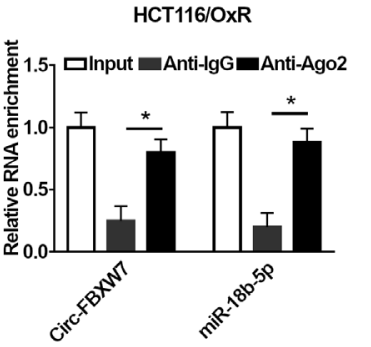


and invasion of SW480/OxR and HCT116/OxR cells by suppressing EMT, reflected by the decrease of $\mathrm{N}$-cad expression but the increase of E-cad expression (Figures 3C, 3D, 3G). Meanwhile, the total intracellular Pt and DNA-bound Pt levels were markedly upregulated in circ-FBXW7-exoincubated SW480/OxR and HCT116/OxR cells (Figures 3E, $3 \mathrm{~F})$. Taken together, these results suggested the intercellular transfer of circ-FBXW7 by exosomes reversed oxaliplatin resistance in CRC cells in vitro.
circ-FBXW7 is a sponge of miR-18b-5p in CRC cells. It has been reported that circRNAs can function as sponges of miRNAs to affect the expression of miRNAs, thus regulating the development of cancers [22]. In this study, miR-18b-5p was found to be elevated in oxaliplatin-resistant CRC patients and resistant CRC cells compared with that in controls (Figures 4A, 4B). Meanwhile, a negative correlation between circ-FBXW7 and miR-18b-5p was observed (Figure 4C). Thus, we assumed that circ-FBXW7 might be a
A
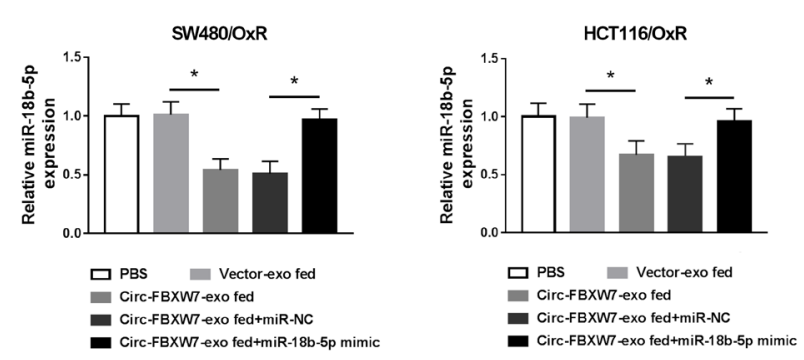

B
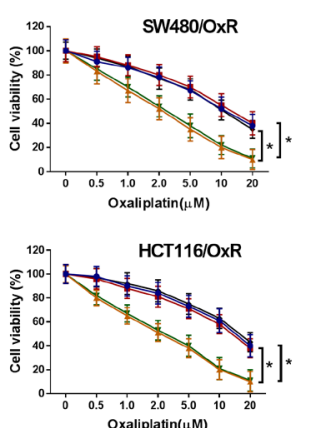

C
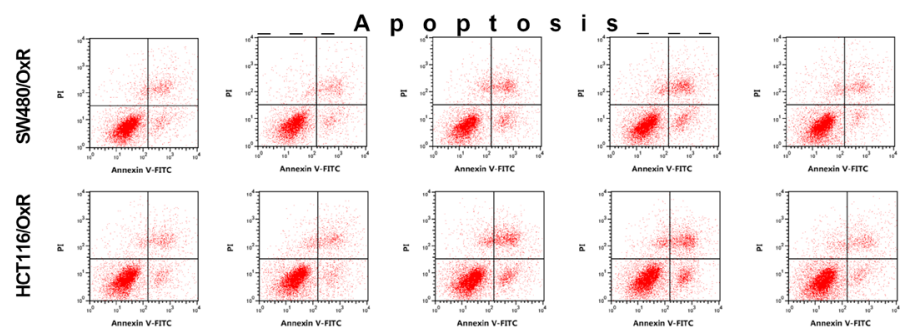

D
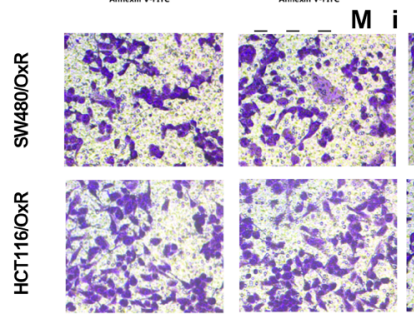

i g $r a t$ i $a n$

E
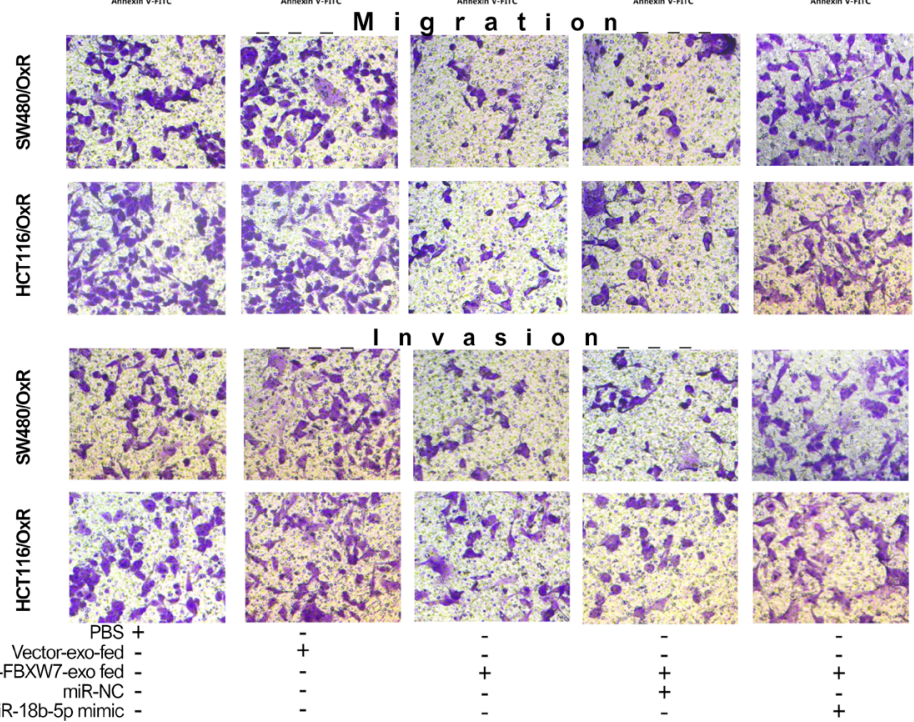

- PBS - Vectorexo fed

7 Circ-FBXW7 exo fed

- Circ-FBXW7-exo fed+miR-18b-5p mimic

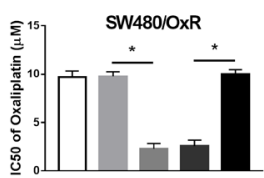

HCT116/OxR
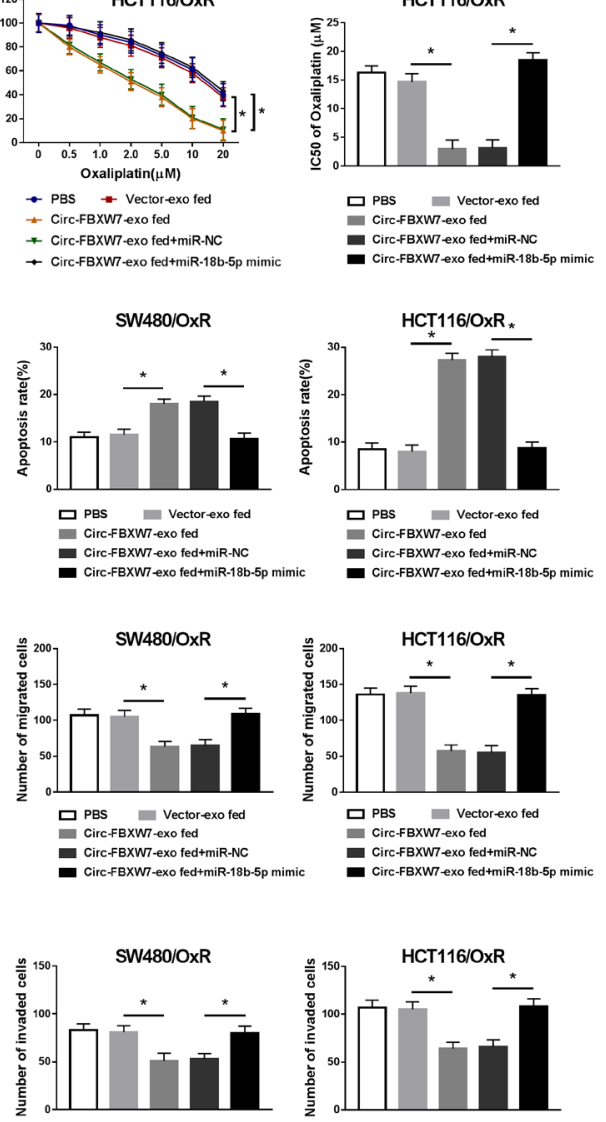

믈 Circ-FXWT-exo fed Circ-BBXWT-exo fedtmir-NC - Circ-FXWT-exo fedtmiR-NC

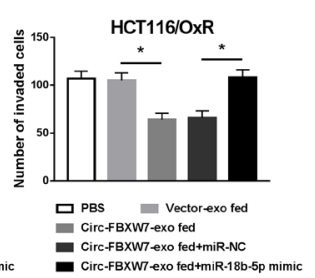

Figure 5. Exosome-mediated transfer of circ-FBXW7 spreads oxaliplatin sensitivity by binding to miR-18b-5p. SW480/OxR and HCT116/OxR cells were transfected with miR-NC or miR-18b-5p mimic after co-cultured with Vector-exo or circ-FBXW7-exo. A) The expression of miR-18b-5p was measured using qRT-PCR in SW480/OxR and HCT116/OxR cells. B) Cell viability was detected using the CCK-8 assay in resistant cells treated with oxaliplatin at indicated concentrations. C) Flow cytometry analysis of resistant cell apoptosis treated with $30 \mu \mathrm{M}$ oxaliplatin was conducted. The transwell assay was used to examine the migration (D) and invasion (E) of SW480/OxR and HCT116/OxR cells. ${ }^{*}$ p $<0.05$ 
sponge of miR-18b-5p in CRC cells. According to the bioinformatics analysis of the starBase 2.0 program, miR-18b-5p was identified to contain the putative binding sites of circFBXW7 (Figure 4D). Subsequently, a dual-luciferase reporter assay was conducted and the reduction of luciferase activity in $293 \mathrm{~T}$ cells co-transfected with circ-FBXW7-WT and miR-18b-5p mimic confirmed the interaction between circFBXW7 and miR-18b-5p (Figure 4E). Furthermore, the RIP assay further verified the direct interaction between circFBXW7 and miR-18b-5p with the significant enrichment of circ-FBXW7 and miR-18b-5p in SW480/OxR and HCT116/ OxR cells after Ago2 RIP, while there were no changes in response to IgG RIP (Figure $4 \mathrm{~F}$ ). These data indicated circFBXW7 directly bound to miR-18b-5p in CRC cells.

Exosome-mediated transfer of circ-FBXW7 spreads oxaliplatin sensitivity by binding to miR-18b-5p. Based on the relationship between circ-FBXW7 and miR-18b-5p, we further investigated whether is circ-FBXW7/miR-18b-5p responsible for the oxaliplatin resistance in CRC cells. SW480/OxR and HCT116/OxR cells were transfected with miR-NC or miR-18b-5p mimic after co-cultured with Vector-exo or circ-FBXW7-exo. Then we found miR-18b-5p was suppressed by exosomal circ-FBXW7 but was rescued by following miR-18b-5p mimic transfection (Figure 5A). After that, results showed the introduction of miR-18b-5p mimic greatly reversed exosomal circ-FBXW7-induced drug sensibility to oxaliplatin (Figure 5B), the promotion of apoptosis
(Figure 5C), the suppression of migration and invasion (Figures 5D, 5E), and the accumulation of Pt (Figures 6A, 6B) in SW480/OxR and HCT116/OxR cells. What's more, western blot analysis suggested that overexpressed miR-18b-5p obviously attenuated exosomal circ-FBXW7mediated inhibition on the protein of MRP-1, MCL-1, and $\mathrm{N}$-cad, as well as the promotion on the protein of cleaved caspase-3 and E-cad in CRC resistant cells (Figure 6C), further indicating miR-18b-5p reversed exosomal circFBXW7-induced drug sensibility to oxaliplatin. In all, these results demonstrated that exosomal circ-FBXW7 could weaken oxaliplatin resistance by sponging $\mathrm{miR}-18 \mathrm{~b}-5 \mathrm{p}$ in CRC resistant cells.

Exosomal circ-FBXW7 suppresses oxaliplatin resistance in vivo. We further detected the effects of exosomal circ-FBXW7 on oxaliplatin-mediated tumor growth in vivo. As shown in Figures 7A and 7B, exosomal circ-FBXW7 inhibited tumor volume and weight of oxaliplatin-resistant mice. Additionally, the expression of circ-FBXW7 was increased whereas miR-18b-5p was decreased in circ-FBXW7-exo tumor tissues (Figure 7C). Besides that, western blot showed the inhibition of MRP-1, MCL-1, and N-cad expression, as well as the promotion of cleaved caspase-3 and E-cad expression in circ-FBXW7-exo oxaliplatin-resistant masses (Figure 7D). Therefore, exosomal circ-FBXW7 ameliorated oxaliplatin resistance in vivo by partially regulating miR-18b-5p.
A
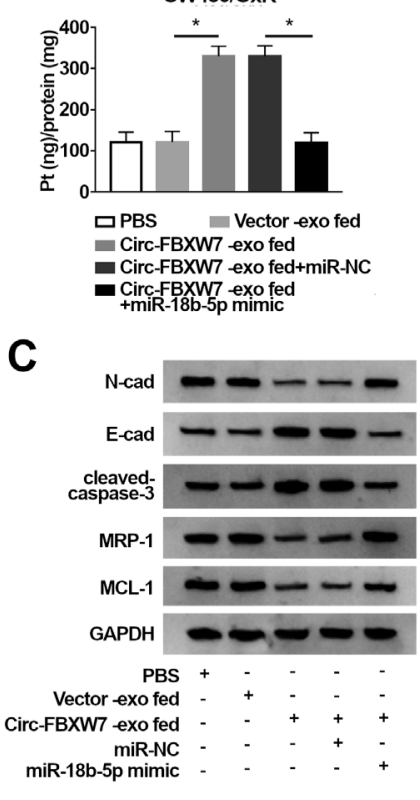
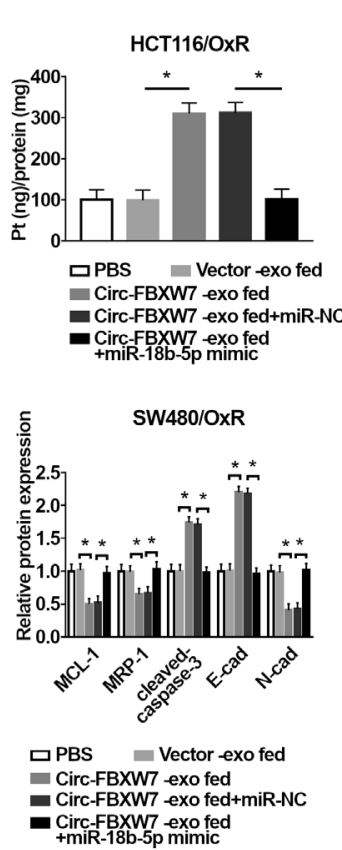

B
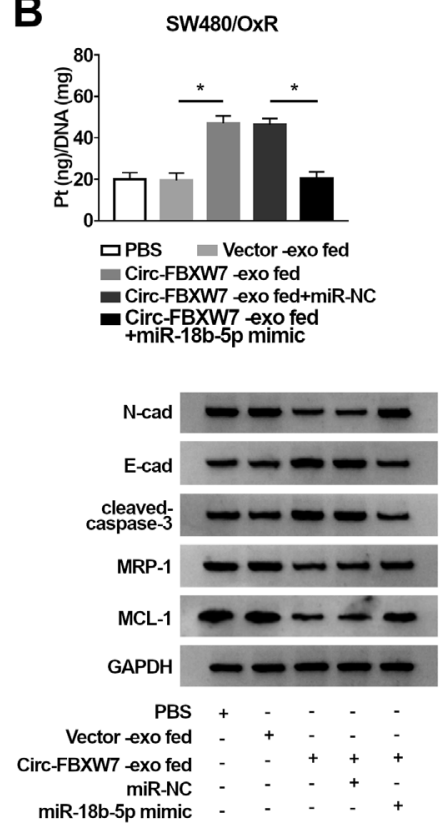
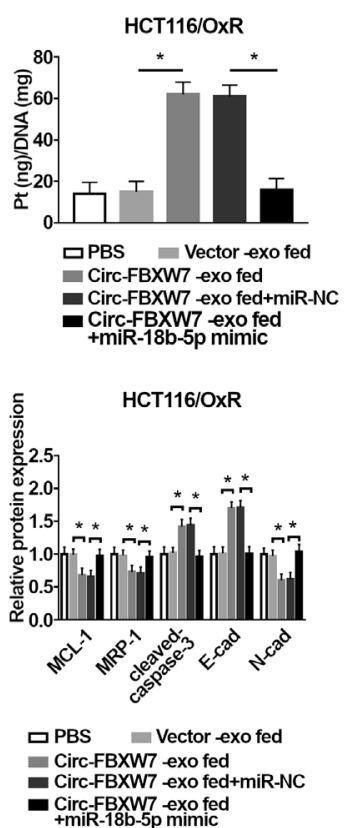

Figure 6. Exosomal circ-FBXW7 weakens the oxaliplatin resistance by sponging miR-18b-5p in CRC resistant cells. A, B) The accumulation of Pt was analyzed in resistant cells after treated with $30 \mu \mathrm{M}$ oxaliplatin for $24 \mathrm{~h}$. C) Western blot was used to measure the level of $\mathrm{N}$-cad, E-cad, cleaved caspase-3, MRP-1, and MCL-1. ${ }^{*} \mathrm{p}<0.05$ 
A

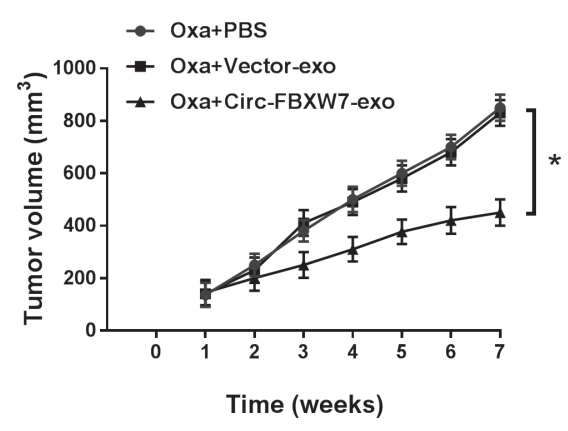

C

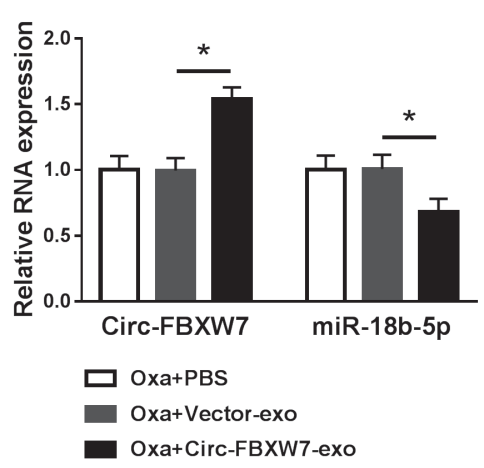

B
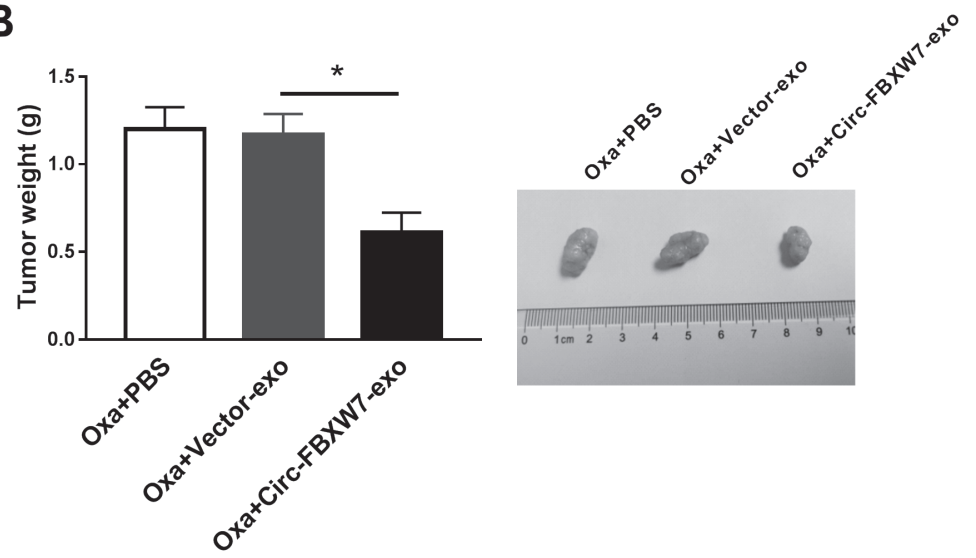

D

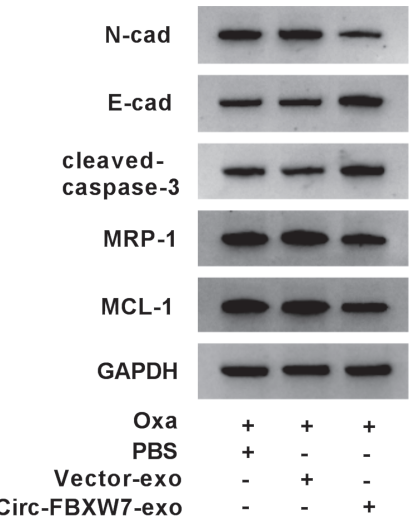

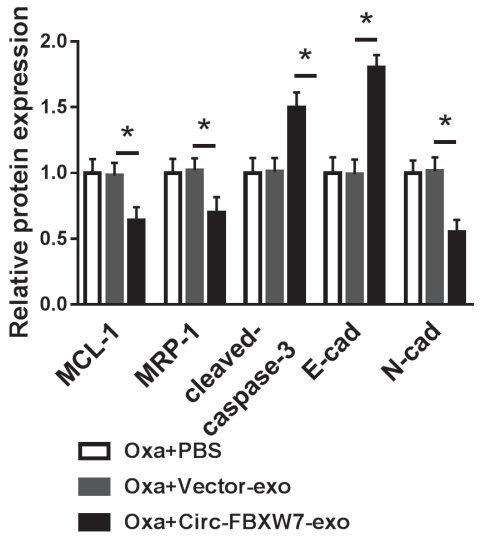

Figure 7. Exosomal circ-FBXW7 suppresses oxaliplatin resistance in vivo. A) Tumor volume was measured every week. B) Tumor weight was measured after 7 weeks. C) The expression of circ-FBXW7 and miR-18b-5p was detected by qRT-PCR. D) Protein expression of MRP-1, MCL-1, Caspases-3, Ecad, and N-cad was evaluated by western blot. ${ }^{*} \mathrm{p}<0.05$

\section{Discussion}

Growing findings suggest that circRNAs play important roles in the homeostasis of cancer cells [23]. Besides that, the implication of circRNAs in relation to chemoresistance in many types of cancers has also been reported. For example, Zhao et al. revealed that circ-Cdrlas ameliorated cisplatin resistance in ovarian cancer by modulating the miR-1270/SCAI network [24]. Liang et al. found out that circ-KDM4C inhibited tumorigenesis and contributed to doxorubicin resistance in breast cancer by regulating PBLD through miR-548p suppression [25]. Luo et al. demonstrated that circRNA_101505 enhanced cisplatin toxicity in hepatocellular carcinoma cells by promoting NOR1 by regulating miR-103 [26]. Thus, circRNAs may be useful markers for chemoresistance.

Up to date, the resistance of chemotherapy in CRC has also become a burden to effectively treat the disease. Nevertheless, some circRNAs have been revealed to associate with the chemoresistance of CRC. For instance, Xiong et al. uncovered that 71 circRNAs were differentially expressed in 5-FU chemoradiation-resistant CRC cells, and among these, circ_0000504 was found to upregulate STAT3 by modulating the miR-485-5p expression, thus promoting chemoradiation-resistance in CRC [27]. In addition, Abu et al. also identified that multiple circRNAs were differentially expressed between chemoresistant and chemosensitive CRC cells to 5-FU and oxaliplatin, and circ_32883 was a promising biotarget for chemoresistant CRC [28]. Therefore, circRNAs are also potential targets to overcome chemoresistance in CRC. Besides that, based on the characteristics of exosomes exchanging their contents in tumor cells and microenvironment [6-8], exosomes have also been considered as promising targets for antitumor therapy in CRC. For example, exosome-transmitted miR-128-3p sensitized oxaliplatin-resistant CRC cells to oxaliplatin [29]. LncRNA CCAL transferred from cancer-related fibroblasts by exosomes accelerated CRC cells resistance to oxaliplatin [30]. Exosomal Wnts derived from cancer-associated fibroblasts inhibited chemosensitivity in CRC [31]. However, large-scale identifications of exosomal circRNA in CRC cell chemoresistance were not yet reported. 
In this study, we found circ-FBXW7 was lowly expressed in oxaliplatin-resistant CRC patients and cells. Subsequently, circ-FBXW7 was confirmed to be secreted by circ-FBXW7transfected FHC cells and could be transferred to resistant CRC cells through exosome secretion. Based on these results, in vitro and in vivo studies were conducted and demonstrated that exosomal circ-FBXW7 led resistant cells to become sensitive to oxaliplatin, increased the oxaliplatin-induced apoptosis, promoted oxaliplatin-mediated EMT inhibition, and suppressed oxaliplatin efflux. Thus, the exosomal transfer of circ-FBXW7 may help to improve the chemosensitivity in CRC.

It has been reported that circRNAs contain multiple types and quantities of miRNAs binding sites, which allow them to specifically sponge to miRNAs to affect miRNAs activity, thus regulating the development of cancers [22]. miR-18b-5p is a functional miRNA. For example, miR-18b-5p acted as a target of ZFPM2-AS1 to be involved in the promotion of cells in lung adenocarcinoma [32]. LncRNA AC073284.4 suppressed cell EMT and migration in breast cancer by directly interacting with miR-18b-5p [33]. Additionally, a recent study indicated miR-18b-5p was highly expressed in CRC and was a potential biomarker for poor prognosis [34]. However, the exact roles of miR-18b-5p in CRC chemoresistance remain unclear. In this study, miR-18b-5p was increased in oxaliplatin-resistant CRC patients and cells. Then using the bioinformatics analysis, miR-18b-5p was identified to be a target of circ-FBXW7. Immediately, the rescue assay showed the exosome-mediated transfer of circ-FBXW7 enhanced oxaliplatin sensitivity by binding to miR-18b-5p in vitro and in vivo.

In conclusion, our study illustrated that exosome-transmitted circ-FBXW7 could ameliorate the chemoresistance to oxaliplatin in CRC cells by sponging miR-18b-5p, indicating a promising therapeutic strategy for the treatment of oxaliplatin-resistant CRC patients.

\section{References}

[1] FERLAY J, SOERJOMATARAM I, DIKSHIT R, ESER S, MATHERS $C$ et al. Cancer incidence and mortality worldwide: sources, methods and major patterns in GLOBOCAN 2012. Int J Cancer 2015; 136: E359-386. https://doi. org/10.1002/ijc.29210

[2] DAY LW, VELAYOS F. Colorectal cancer screening and surveillance in the elderly: updates and controversies. Gut Liver 2015; 9: 143-151. https://doi.org/10.5009/gnl14302

[3] BRODY H. Colorectal cancer. Nature 2015; 521: S1. https:// doi.org/10.1038/521S1a

[4] LI L, SHANG J, ZHANG Y, LIU S, PENG Y et al. MEG3 is a prognostic factor for CRC and promotes chemosensitivity by enhancing oxaliplatin-induced cell apoptosis. Oncol Rep 2017; 38: 1383-1392. https://doi.org/10.3892/or.2017.5828
[5] COCUCCI E, MELDOLESI J. Ectosomes and exosomes: shedding the confusion between extracellular vesicles. Trends Cell Biol 2015; 25: 364-372. https://doi.org/10.1016/j. tcb.2015.01.004

[6] ABELS ER, BREAKEFIELD XO. Introduction to Extracellular Vesicles: Biogenesis, RNA Cargo Selection, Content, Release, and Uptake. Cell Mol Neurobiol 2016; 36: 301-312. https://doi.org/10.1007/s10571-016-0366-Z

[7] CHOI DS, KIM DK, KIM YK, GHO YS. Proteomics, transcriptomics and lipidomics of exosomes and ectosomes. Proteomics 2013; 13: 1554-1571. https://doi.org/10.1002/ pmic.201200329

[8] LI Y, ZHENG Q, BAO C, LI S, GUO W et al. Circular RNA is enriched and stable in exosomes: a promising biomarker for cancer diagnosis. Cell Res 2015; 25: 981-984. https://doi. org/10.1038/cr.2015.82

[9] MULLER L, MITSUHASHI M, SIMMS P, GOODING WE, WHITESIDE TL. Tumor-derived exosomes regulate expression of immune function-related genes in human $\mathrm{T}$ cell subsets. Sci Rep 2016; 6: 20254. https://doi.org/10.1038/ srep20254

[10] ZHAO H, YANG L, BADDOUR J, ACHREJA A, BERNARD V et al. Tumor microenvironment derived exosomes pleiotropically modulate cancer cell metabolism. Elife 2016; 5: e10250. https://doi.org/10.7554/eLife.10250

[11] AZMI AS, BAO B, SARKAR FH. Exosomes in cancer development, metastasis, and drug resistance: a comprehensive review. Cancer Metastasis Rev 2013; 32: 623-642. https://doi. org/10.1007/s10555-013-9441-9

[12] YANG T, MARTIN P, FOGARTY B, BROWN A, SCHURMAN K et al. Exosome delivered anticancer drugs across the blood-brain barrier for brain cancer therapy in Danio rerio. Pharm Res 2015; 32: 2003-2014. https://doi.org/10.1007/ s11095-014-1593-y

[13] URBANELLI L, BURATTA S, SAGINI K, FERRARA G, LANNI $M$ et al. Exosome-based strategies for diagnosis and therapy. Recent Pat CNS Drug Discov 2015; 10: 10-27. https://doi.org/10.2174/1574889810666150702124059

[14] RYBAK-WOLF A, STOTTMEISTER C, GLAZAR P, JENS $\mathrm{M}, \mathrm{PINO} \mathrm{N}$ et al. Circular RNAs in the Mammalian Brain Are Highly Abundant, Conserved, and Dynamically Expressed. Mol Cell 2015; 58: 870-885. https://doi.org/10.1016/j.molcel.2015.03.027

[15] ZHAO ZJ, SHEN J. Circular RNA participates in the carcinogenesis and the malignant behavior of cancer. RNA Biol 2017; 14: 514-521. https://doi.org/10.1080/15476286.2015.1 122162

[16] CHEN Q, LIU T, BAO Y, ZHAO T, WANG J et al. CircRNA cRAPGEF5 inhibits the growth and metastasis of renal cell carcinoma via the miR-27a-3p/TXNIP pathway. Cancer Lett 2020; 469: 68-77. https://doi.org/10.1016/j.canlet.2019.10.017

[17] LI R, WU B, XIA J, YE L, YANG X. Circular RNA hsa_circRNA_102958 promotes tumorigenesis of colorectal cancer via miR-585/CDC25B axis. Cancer Manag Res 2019; 11: 6887-6893. https://doi.org/10.2147/CMAR.S212180 
[18] YANG G, ZHANG T, YE J, YANG J, CHEN C et al. Circ-ITGA7 sponges miR-3187-3p to upregulate ASXL1, suppressing colorectal cancer proliferation. Cancer Manag Res 2019; 11: 6499-6509. https://doi.org/10.2147/CMAR.S203137

[19] LU H, YAO B, WEN X, JIA B. FBXW7 circular RNA regulates proliferation, migration and invasion of colorectal carcinoma through NEK2, mTOR, and PTEN signaling pathways in vitro and in vivo. BMC Cancer 2019; 19: 918. https:// doi.org/10.1186/s12885-019-6028-Z

[20] YANG AD, FAN F, CAMP ER, VAN BUREN G, LIU W et al. Chronic oxaliplatin resistance induces epithelial-to-mesenchymal transition in colorectal cancer cell lines. Clin Cancer Res 2006; 12: 4147-4153. https://doi.org/10.1158/1078-0432. CCR-06-0038

[21] Melo SA, Sugimoto H, O'Connell JT, Kato N., Villanueva A et al. Cancer exosomes perform cell-independent microRNA biogenesis and promote tumorigenesis. Cancer Cell 2014; 26: 707-721. https://doi.org/10.1016/j.ccell.2014.09.005

[22] HE R, LIU P, XIE X, ZHOU Y, LIAO Q et al. circGFRA1 and GFRA1 act as ceRNAs in triple negative breast cancer by regulating miR-34a. J Exp Clin Cancer Res 2017; 36: 145. https://doi.org/10.1186/s13046-017-0614-1

[23] QU S, YANG X, LI X, WANG J, GAO Y et al. Circular RNA: A new star of noncoding RNAs. Cancer Lett 2015; 365: 141148. https://doi.org/10.1016/j.canlet.2015.06.003

[24] ZHAO Z, JI M, WANG Q, HE N, LI Y. Circular RNA Cdrlas Upregulates SCAI to Suppress Cisplatin Resistance in Ovarian Cancer via miR-1270 Suppression. Mol Ther Nucleic Acids 2019; 18: 24-33. https://doi.org/10.1016/j.omtn.2019.07.012

[25] LIANG Y, SONG X, LI Y, SU P, HAN D et al. circKDM4C suppresses tumor progression and attenuates doxorubicin resistance by regulating miR-548p/PBLD axis in breast cancer. Oncogene 2019; 38: 6850-6866. https://doi.org/10.1038/ s41388-019-0926-Z

[26] LUO Y, FU Y, HUANG R, GAO M, LIU F et al. CircRNA_101505 sensitizes hepatocellular carcinoma cells to cisplatin by sponging miR-103 and promotes oxidored-nitro domain-containing protein 1 expression. Cell Death Discov 2019; 5: 121. https://doi.org/10.1038/s41420-019-0202-6
[27] XIONG W, AI YQ, LI YF, YE Q, CHEN ZT et al. Microarray Analysis of Circular RNA Expression Profile Associated with 5-Fluorouracil-Based Chemoradiation Resistance in Colorectal Cancer Cells. Biomed Res Int 2017; 2017: 8421614. https://doi.org/10.1155/2017/8421614

[28] ABU N, HON KW, JEYARAMAN S, YAHAYA A, ABDULLAH NM et al. Identification of differentially expressed circular RNAs in chemoresistant colorectal cancer. Epigenomics 2019; 11: 875-884. https://doi.org/10.2217/epi-2019-0042

[29] LIU T, ZHANG X, DU L, WANG Y, LIU X et al. Exosometransmitted miR-128-3p increase chemosensitivity of oxaliplatin-resistant colorectal cancer. Mol Cancer 2019; 18: 43. https://doi.org/10.1186/s12943-019-0981-7

[30] DENG X, RUAN H, ZHANG X, XU X, ZHU Y et al. Long noncoding RNA CCAL transferred from fibroblasts by exosomes promotes chemoresistance of colorectal cancer cells. Int J Cancer 2020; 146: 1700-1716. https://doi.org/10.1002/ ijc.32608

[31] HU YB, YAN C, MU L, MI YL, ZHAO H et al. Exosomal Wnt-induced dedifferentiation of colorectal cancer cells contributes to chemotherapy resistance. Oncogene 2019; 38: 1951-1965. https://doi.org/10.1038/s41388-018-0557-9

[32] XUE M, TAO W, YU S, YAN Z, PENG Q et al. lncRNA ZFPM2-AS1 promotes proliferation via miR-18b-5p/VMA21 axis in lung adenocarcinoma. J Cell Biochem 2020; 121: 313-321. https://doi.org/10.1002/jcb.29176

[33] WANG YY, YAN L, YANG S, XU HN, CHEN TT et al. Long noncoding RNA AC073284.4 suppresses epithelialmesenchymal transition by sponging miR-18b-5p in paclitaxel-resistant breast cancer cells. J Cell Physiol 2019; 234: 23202-23215. https://doi.org/10.1002/jcp.28887

[34] ZHANG H, ZHU M, SHAN X, ZHOU X, WANG T et al. A panel of seven-miRNA signature in plasma as potential biomarker for colorectal cancer diagnosis. Gene 2019; 687: 246-254. https://doi.org/10.1016/j.gene.2018.11.055 